Note

\title{
GC/MS (SIM) Determination of 14 Pesticides Including Cyhexatin and 2,4,5-T in Nuts
}

\author{
(Received September 30, 1996) \\ Masaru Kawasaki, Katsuharu Fukuhara, Rieko Katsumura,
Noriko Takasaka and Sadao Uchiyama \\ (Hatano Research Institute Food \& Drug Safety Center: \\ 729-5, Ochiai, Hadano, Kanagawa 257, Japan)
}

\begin{abstract}
GC/MS with selected ion monitoring ((GC/MS (SIM)) has been developed as a screening method for the determination of 14 pesticides residues including cyhexatin and 2,4,5-T in nuts. The pesticides were extracted with acetone from nuts, and then partitioned between $n$-hexane and acetonitrile. Clean-up was conducted by solid-phase extraction (SPE) with a Bond Elut Florisil column. Most of the pesticides were eluted in a mixture of $n$-hexane and acetone (17: 3) from the column, and the eluate was examined by GC/MS (SIM). Cyhexatin and 2,4,5-T were analyzed after derivatization and SPE clean-up. Recoveries of 14 pesticides from 6 kinds of nuts were mostly over $80 \%$. This method is effective for multi-residue analysis of pesticides in nuts.
\end{abstract}

Key words: gas chromatography-mass spectrometry (GC/MS); MS fragmentation; pesticide; solid-phase extraction (SPE); selected ion monitoring (SIM); cyhexatin; 2,4,5-T

\section{Introduction}

Much attention has been paid to pesticide residues in foods in Japan, particularly because of the increase in imported foods. The Ministry of Health and Welfare in Japan has specified the residue levels for 138 pesticides in the Law of Food Sanitation up to $1996^{1-6)}$. Several GC/MS methods for newly specified pesticides have been reported recently ${ }^{7)-10}$. The feasibility of multi-residue analyses of 53 of these 138 pesticides by a GC/MS (SIM) method was discussed in our previous paper ${ }^{11}$. This paper deals with a study of multiresidue analysis with GC/MS (SIM) for 14 pesticides, including cyhexatin and 2,4,5-T, and with clean-up by the solid-phase extraction (SPE) from nuts, which contain oily components that are expected to disturb the analyses.

\section{Materials and Methods}

\section{Solvents and reagents}

Organic solvents such as acetone, $n$-hexane and acetonitrile were of pesticide residue analysis grade. Reagents were of the same grade or specific grade.

\section{Pesticide standards}

Standard reference materials of 14 pesticides, chlorfenvinphos (mixture of $Z$ and $E$ ), cyfluthrin (mixture of isomers), cyhexatin, fenobucarb, fenvalerate, lenacil, methiocarb, myclobutanil, phoxim, pirimiphos-methyl, propiconazole, pyridaben, thiometon and 2,4,5-T, were purchased from Hayashi Pure Chemical Industries, Ltd. (Osaka, Japan) or Wako Pure Chemical Industries, Ltd. (Osaka, Japan).

\section{Pesticide standard solutions}

Pesticide standard solutions $(1000 \mu \mathrm{g} / \mathrm{mL})$ were prepared by dissolving the pesticide in acetone.

\section{Spiking solution}

Spiking solution (each concentration: $2 \mu \mathrm{g} /$ $\mathrm{mL}$ ) was prepared by combining 14 pesticide 
standard solutions and diluting the mixture with acetone.

\section{Solid phase extraction (SPE) column}

Bond Elut LRC ( $3 \mathrm{cc} / 500 \mathrm{MG})$ Florisil purchased from Varian Co. (U.S.A.) was used for solid phase extraction (SPE).

\section{Samples}

Macadamia nuts, hazel nuts, cashew nuts, pistachio nuts, peanuts and pine nuts were obtained from commercial sources in Japan.

\section{Apparatus}

Gas chromatograph-mass spectrometer: Shimadzu GC-17A gas chromatograph coupled with a Parvum QP 5000 mass spectrometer and a Shimadzu AOC-17 injector.

Data processing apparatus: Sanyo Co. AXAGE $\nu$ computer, Shimadzu operating system software class 5000, and MS-Windows.

GC conditions: GC column, J \& W Scientific capillary column DB- $5 \mathrm{~ms}(0.25 \mathrm{~mm}$ i.d. $\times 30 \mathrm{~m}$, film thickness $0.25 \mu \mathrm{m})$. Oven temp., $50^{\circ} \mathrm{C}(1.5$ $\min ) \rightarrow 300^{\circ} \mathrm{C}(10 \mathrm{~min})$, increased at $10^{\circ} \mathrm{C} / \mathrm{min}$. Injection temp., $250^{\circ} \mathrm{C}$; transfer line temp., $280^{\circ} \mathrm{C}$; carrier gas, He at $20 \mathrm{~mL} / \mathrm{min}$; injection volume, $1 \mu \mathrm{L}$ (splitless); sampling time, $1.00 \mathrm{~min}$.

$M S$ conditions: Electron impact ionization (EI); ion source voltage, $70 \mathrm{eV}$; detector gain, $1.75 \mathrm{kV}$; solvent elution time, $3.00 \mathrm{~min}$; measurement time, $4.00-35.00 \mathrm{~min}$; mass range, $\mathrm{m} / z$ 40.00400.00 .

\section{Selected ion monitoring (SIM) method}

SIM operating conditions were as follows. Detector gain, 1.75 or $2.5 \mathrm{kV}$; maximum ion set number, 20; solvent elution time, $3.00 \mathrm{~min}$; sampling rate, $0.30 \mathrm{sec}$; micro scan width: 0.50 . GC/ MS operating software is class 5000 (Shimadzu), with the Windows 3.0 operating system.

Investigation of solid phase extraction (SPE) elution pattern

A Bond Elut Florisil column was conditioned with $2 \mathrm{~mL}$ of $n$-hexane, then $2 \mathrm{~mL}$ aliquots of standard solutions were applied to the column, and eluted with $5 \mathrm{~mL}$ of $n$-hexane (Fraction 1), 5 $\mathrm{mL}$ of $n$-hexane-acetone (17:3) (Fraction 2) and $5 \mathrm{~mL}$ of $n$-hexane-acetone (1:1) (Fraction 3 ).
Each eluate was evaporated under $\mathrm{N}_{2}$ gas, and the residue was dissolved in $2 \mathrm{~mL}$ of $n$-hexane. The solution was measured by GC/MS (SIM) to obtain the SPE elution pattern.

\section{Preparation of the test solution}

Pesticides except cyhexatin and 2,4,5-T were extracted twice from ground nuts $(20 \mathrm{~g})$ with $100 \mathrm{~mL}$ portions of acetone. After paper filtration, the combined extract was evaporated under reduced pressure to remove acetone. The residual aqueous solution was mixed with 100 $\mathrm{mL}$ of saturated $\mathrm{NaCl}$ solution and further extracted with two $100 \mathrm{~mL}$ portions of $n$-hexane. The $n$-hexane layers were combined and then dehydrated with anhydrous $\mathrm{Na}_{2} \mathrm{SO}_{4}$. The $n$ hexane solution was evaporated to a small volume and dried with $\mathrm{N}_{2}$ gas. The residue was partitioned between two $30 \mathrm{~mL}$ portions of $n$ hexane-saturated $\mathrm{MeCN}$ and $30 \mathrm{~mL}$ of $\mathrm{MeCN}-$ saturated hexane. The MeCN layers were combined and evaporated to dryness with $\mathrm{N}_{2}$ gas. The residue was dissolved in $20 \mathrm{~mL}$ of $n$-hexane. A $5 \mathrm{~mL}$ aliquot of the $n$-hexane solution was subjected to SPE clean-up as described above. Test solutions of cyhexatin and 2,4,5-T were prepared according to the Japanese standard methods $^{4}$ and then subjected to SPE as described above. Fraction $1(5 \mathrm{~mL})$ is for analysis of cyhexatin derivative and fraction $2(5 \mathrm{~mL})$ for analysis of other pesticides.

\section{Recovery test}

Fourteen pesticides were spiked in 6 kinds of nuts at the concentration of $0.2 \mathrm{ppm}$. Each recovery was obtained as the average of three trials $(n=3)$.

\section{Results and Discussion}

In this experiment, three steps of extraction with organic solvent, clean-up by solid-phase extraction (SPE) and GC/MS (SIM) were adopted for a multiresidue analysis of pesticides in oily nuts.

\section{Extraction and clean-up with SPE}

Extraction of nuts with acetone, and partition between $n$-hexane and MeCN were useful for the purification of pesticides from oily nut samples. Further clean-up was conducted by the SPE 


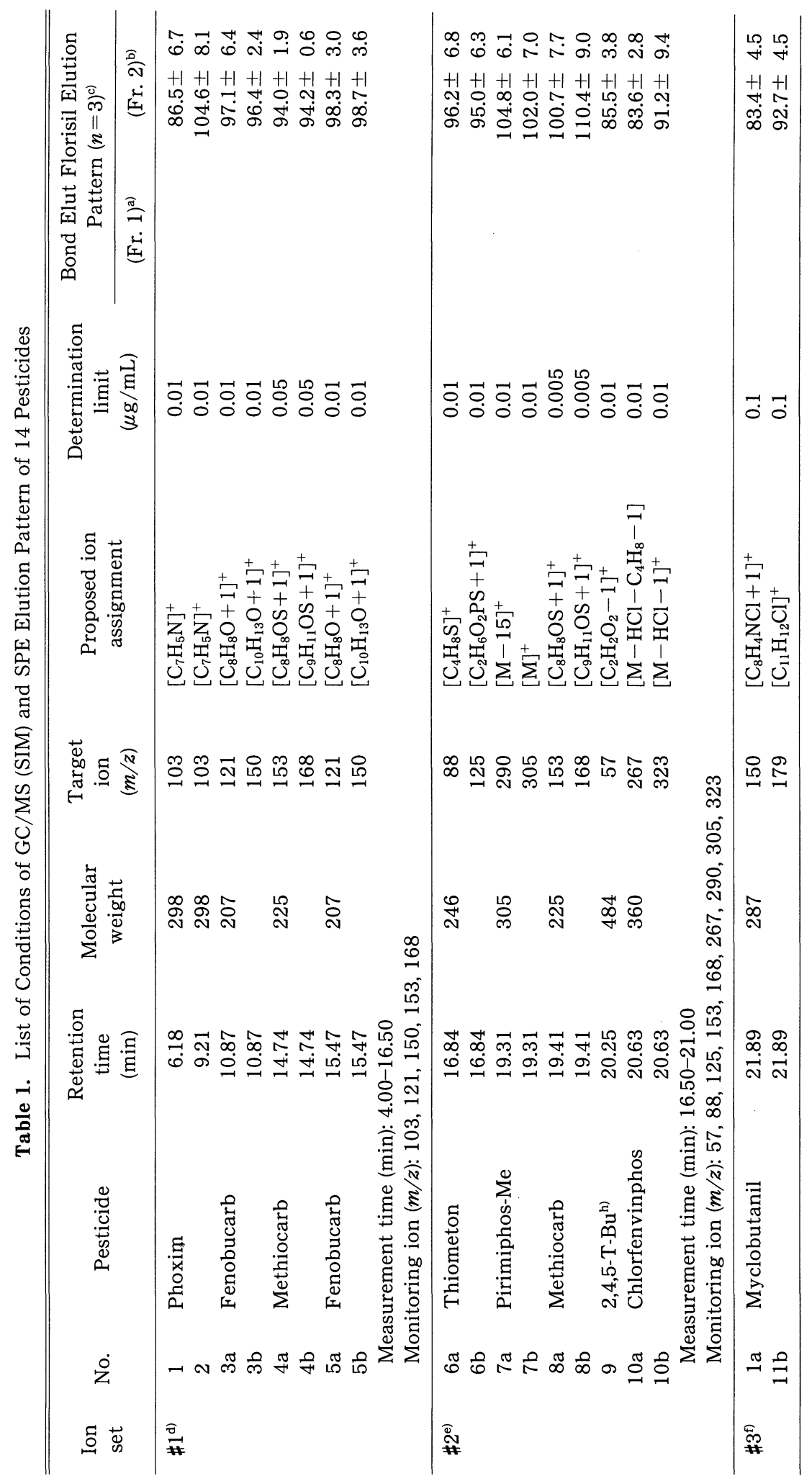




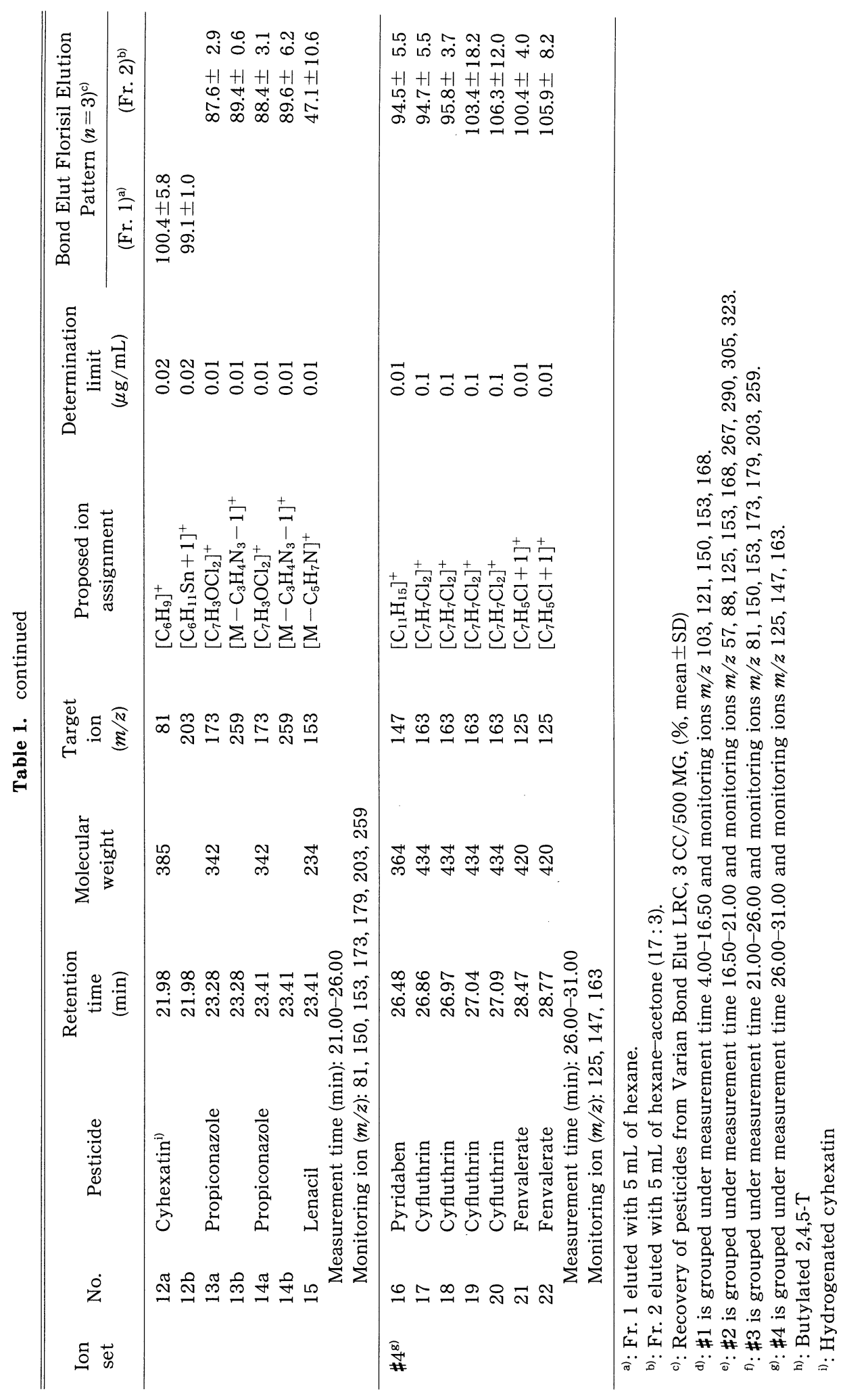


method. The SPE with a Bond Elut-Florisil column gave good separation and recoveries of pesticides $^{11}$. In the present experiment, we adopted the same column and eluant system. As described in experimental, the pesticides were eluted in three fractions. Cyhexatin and 2,4,5-T were subjected to SPE after derivatization. Most of the pesticides were eluted in Fr. 2 (nhexane-acetone $(17: 3))$, and the cyhexatin derivative was eluted in Fr. 1 ( $n$-hexane). No pesticides were observed in Fr. 3.

Recoveries of most of these pesticides through the SPE column were over $80 \%$, but that of lenacil was low (47.1\%). Lenacil is well known to be adsorbed on a Florisil column ${ }^{12)}$ and to show poor recovery from the column. 2,4,5-T and cyhexatin derivatives gave good recoveries by SPE. Thus, SPE appears to be useful for the clean-up of pesticides except lenacil.

\section{Target ions in $G C / M S$}

$\mathrm{GC} / \mathrm{MS}$ of pesticides were examined to select the optimum fragment ions for identification of pesticides. GC/MS of cyhexatin and 2,4,5-T were taken after derivatization. The structures of major fragment ions were determined by well established methods ${ }^{11)}$ of MS analysis, and target ions for characterizing each pesticide were selected as shown in Table 1.

\section{3. $G C / M S$ (SIM) method}

Most of the pesticides were measured by the GC/MS (SIM) method using the target ions in Table 1, which gives the retention time, target ions $(m / z)$ and proposed ion assignments. Most of the pesticides afforded two suitable target ions.

Fourteen pesticides were grouped into 4 ion sets $(\# 1-\# 4)$ for effective SIM measurement on the basis of retention time. Most of the 14 pesticides were detectable by the GC/MS (SIM) at levels of $1 / 5$ to $1 / 10$ of the maximum residue limits permitted by the Japanese Law of Food Sanitation.

Under the experimental conditions, the retention time of propiconazole was very close to that of lenacil, and they could not be separated on the total ion chromatogram (TIC). However, when two target ions of $m / z 173$ and 259 for propiconazole and one ion of $m / z 153$ for lenacil were chosen, as shown in Fig. $1(\$ 3)$, they could be measured separately. A single base peak was observed in the MS of lenacil, so one target ion $(m / z 153)$ was selected.

Thus, 14 pesticides including cyhexatin and $2,4,5$ - $T$ could be analyzed at the same time with high sensitivity by this method.

Standard solutions in hexane were prepared at three different concentrations ranging from 0.01 to $0.2 \mu \mathrm{g} / \mathrm{mL}$. Linear least-squares regression analysis was used and every pesticide showed good linearities. Determination limits of pesticides were in the range of 0.005 to $0.1 \mu \mathrm{g} /$ $\mathrm{mL}$ as described in Table 1. The total ion chromatograms for the standard solution and macadamia nuts extract are shown in Fig. 2. No interfering peaks were observed in any sample solution.

Although cyfulthrin, cyhexatin, phoxim and methiocarb have been analyzed by GC-ECD, GC-FPD or HPLC as Japanese official methods, $\mathrm{GC} / \mathrm{MS}$ (SIM) is also available for the analyses of these pesticides. We are currently attempting to combine the SIM approach for the 53 pesticides reported in the previous paper ${ }^{11)}$ with that for the 14 pesticides in this paper.

\section{Recoveries}

Pesticides equivalent to $0.2 \mathrm{ppm}$ were spiked in $20 \mathrm{~g}$ of homogenized samples. The recoveries from nuts are shown in Table 2. Most of the pesticides except thiometon in pistachio nuts gave good recoveries (80-105\%). The recovery of thiometon for pistachio nuts was low (26.8, $30.6 \%$ ). Although the reason for this is unclear, it may be related to the abundance of chlorophyll in pistachio nuts. On the other hand, lenacil dissolved in $n$-hexane showed a low recovery from the SPE column, but, as shown in Table 2 it gave high recovery from nuts (87.8$100.8 \%$ ). Lenacil may be well eluted from the column in the presence of certain components in nuts. Further study is necessary on the recovery of pesticides from various foods, especially on the effects of food components on the affinity of the Florisil SPE column for pesticides. 

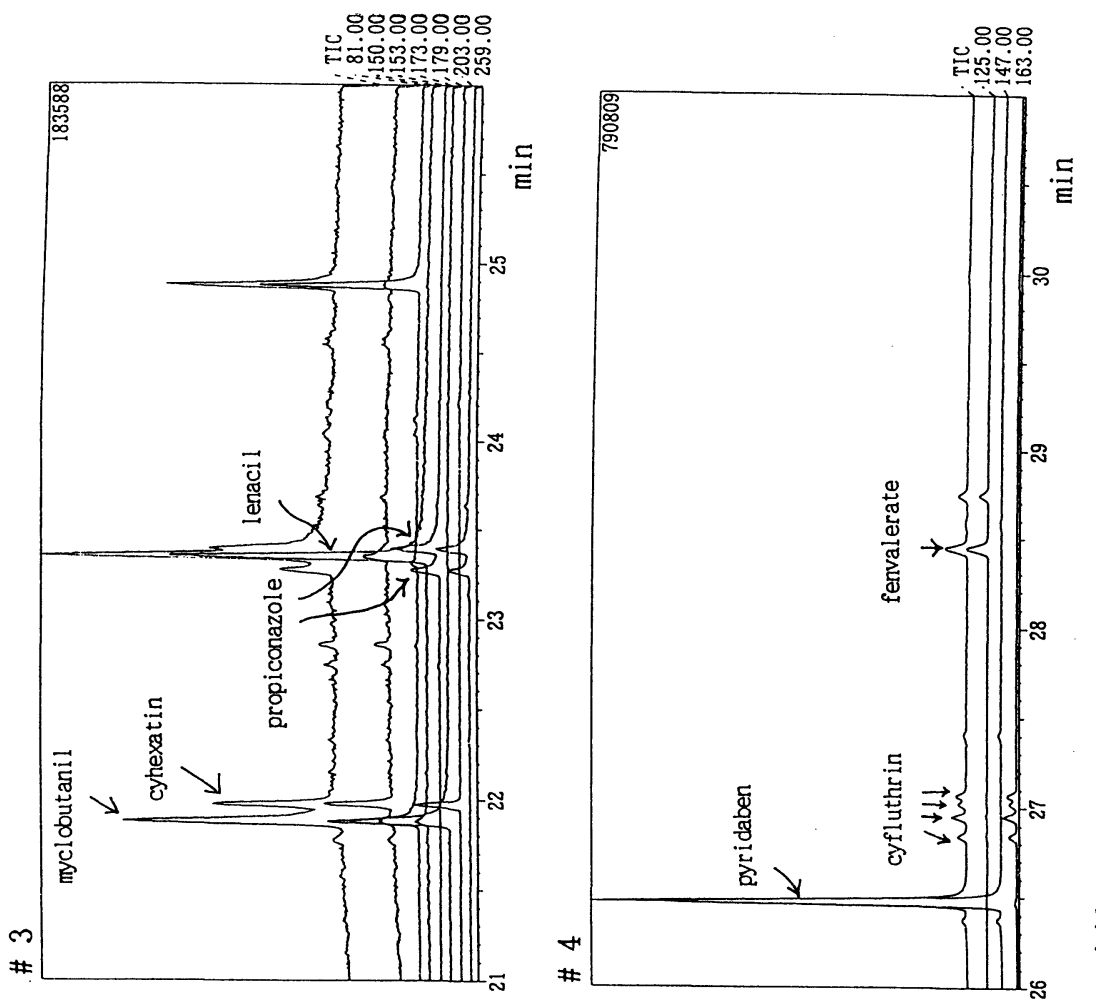

듵
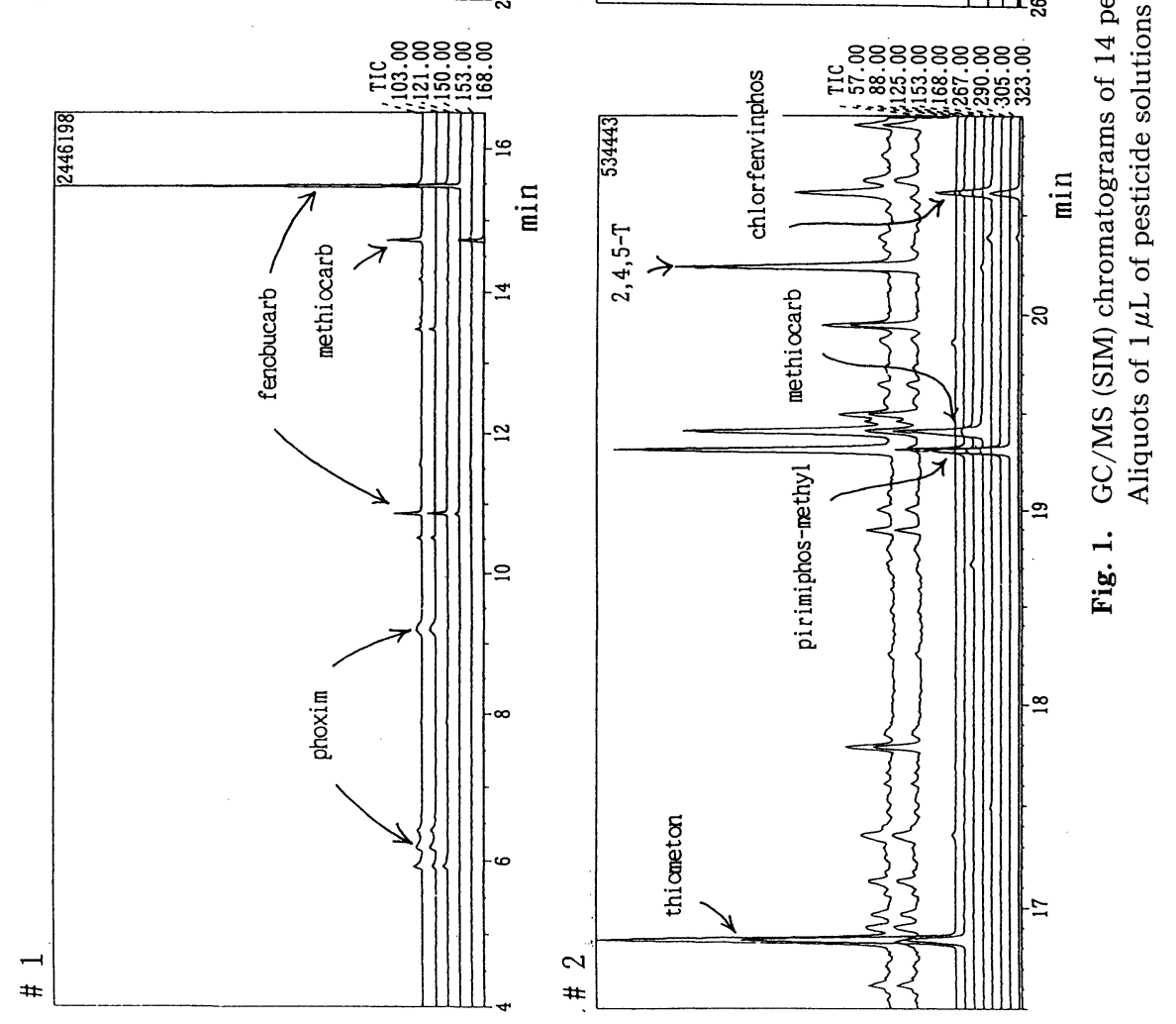

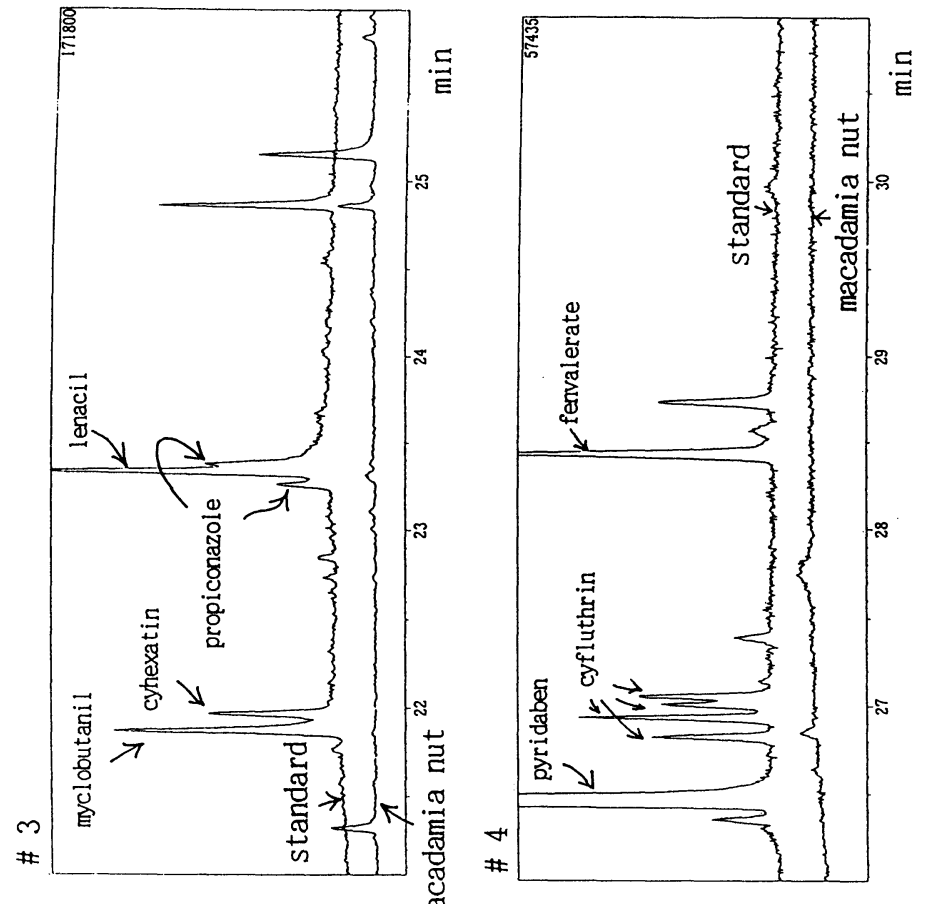

星
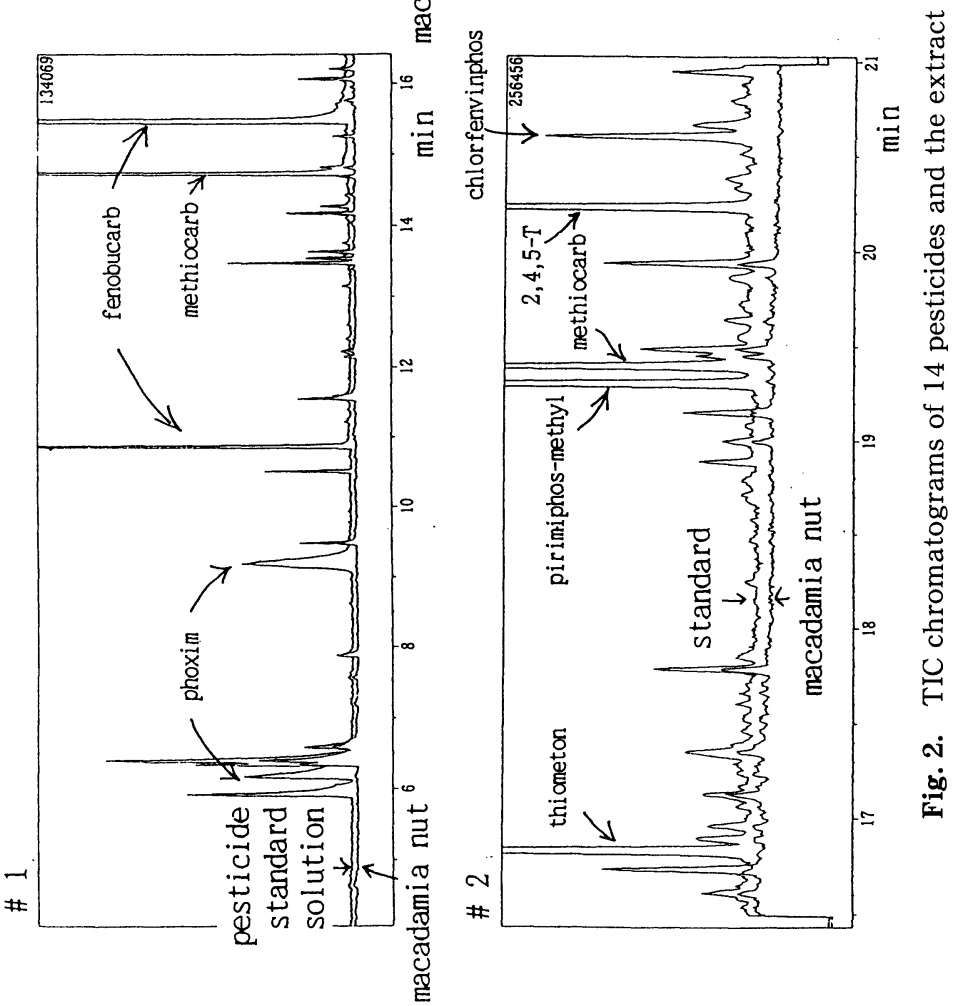


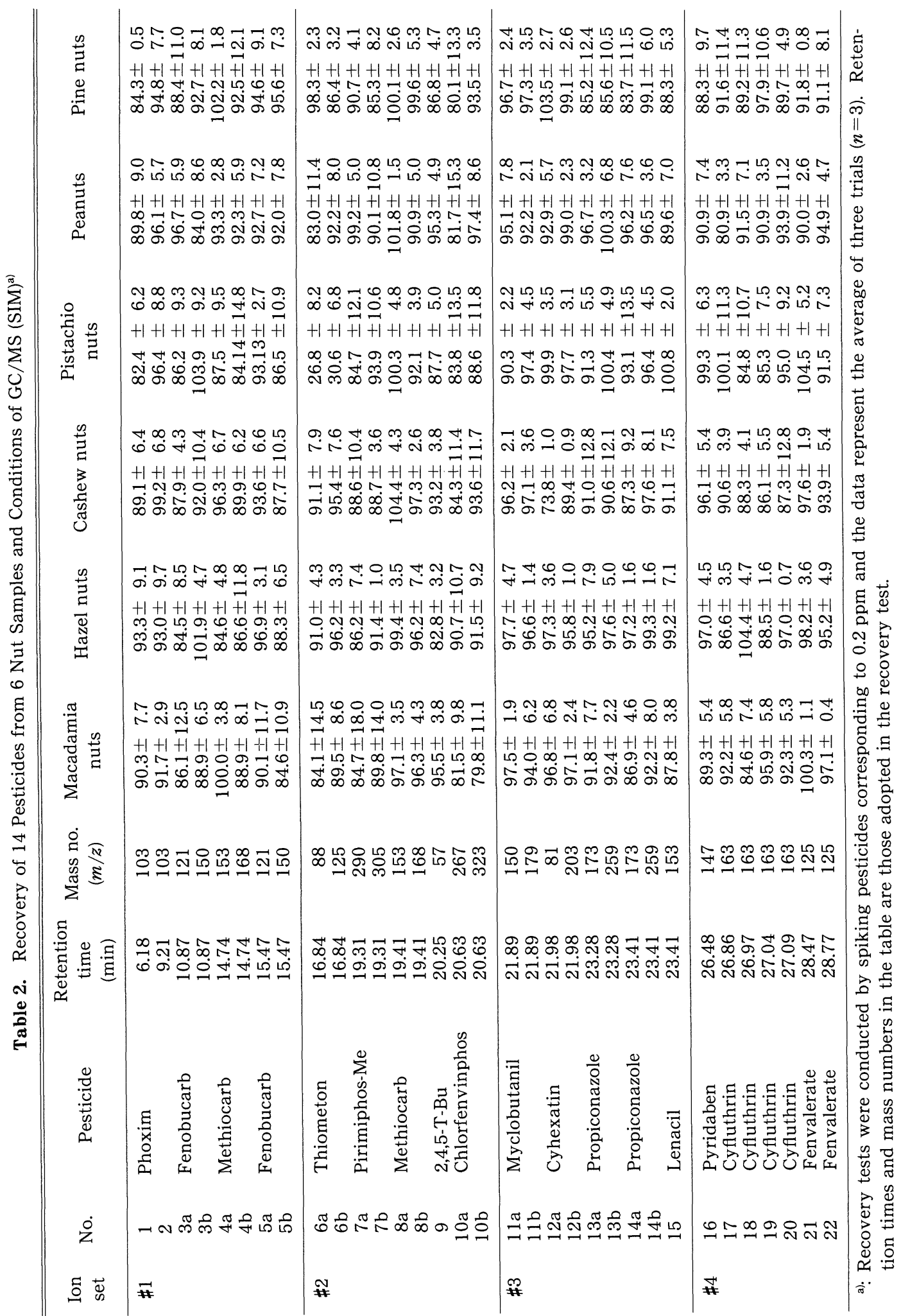




\section{Conclusions}

1. Multi-residue analysis of 14 pesticides was studied by a combination of GC/MS (SIM) and SPE.

2. Most of the 14 pesticides had high recoveries (over $80 \%$ ) from 6 kinds of nuts except thiometon from pistachio nuts.

3. Clean-up with SPE is available for cyhexatin and 2,4,5-T derivatives.

4. Cyfulthrin, cyhexatin, phoxim and methiocarb were analyzed more specifically by the GC/ MS (SIM) method than by the Japanese official methods.

\section{References}

1) Ministry of Health and Welfare of Japan: Bulletin 239, Oct. 27 (1992).

2) Ministry of Health and Welfare of Japan: Bulletin 68, Mar. 4 (1993).

3) Ministry of Health and Welfare of Japan: Bulletin 200, Sep. 14 (1993).
4) Ministry of Health and Welfare of Japan: Bulletin 199, Jun. 9 (1994).

5) Ministry of Health and Welfare of Japan: Bulletin 153, Aug. 14 (1995).

6) Ministry of Health and Welfare of Japan: Bulletin 198, Sep. 2 (1996).

7) Nemoto, S., Shimizu, R., Kikawa, H., Sasaki, K., Saito, Y.: J. Food Hyg. Soc. Japan 36, 233-243 (1995).

8) Akiyama, Y., Takeda, N., Adachi, K.: ibid. 36, 42-49 (1995).

9) Miyata, M., Hirahara, Y., Narita, M., Kimura, M., Watanabe, Y., Ito, S., Takeda, H., Kobayashi, A., Tonogai, Y., Nakamura, Y., Tsumura, Y., Shibata, T.: ibid. 37, 158-164 (1995).

10) Fillion, J., Hindle, R., Lacroix, M., Selwyn, J.: J. AOAC Int. 78, 1,252-1,266 (1995).

11) Kawasaki, M., Fukuhara, K., Uchiyama, S.: J. Food Hyg. Soc. Japan 35, 479-496 (1994).

12) Goto, S., Kato, S.: "Analytical Methods of Pesticides Residues Addendum" p. 319-320 (1988) Soft Scientific Co., Tokyo. 